\title{
MULTIDRUG-RESISTANCE PATTERNS AND DETECTION OF PSTS GENE IN CLINICAL ISOLATES OF PSEUDOMONAS AERUGINOSA FROM NSUKKA, SOUTHEAST NIGERIA
}

\author{
MARTINA C AGBO ${ }^{1 *}$, IFEOMA M EZEONU ${ }^{2}$, ANTHONY C IKE ${ }^{2}$, CELESTINA C UGWU ${ }^{3}$
}

${ }^{1}$ Department of Pharmaceutical Microbiology and Biotechnology, University of Nigeria, Nsukka, Nigeria. ${ }^{2}$ Department of Microbiology, University of Nigeria, Nsukka, Nigeria. ${ }^{3}$ Department of Applied Microbiology and Brewing, Enugu State University of Science and Technology, Enugu, Nigeria. Email: martina.agbo@unn.edu.ng

Received: 20 December 2019, Revised and Accepted: 20 February 2020

ABSTRACT

Objective: This study was aimed to determine the antibiotic resistance patterns of clinical Pseudomonas aeruginosa isolates and to detect the presence of $P s t S$ gene.

Methods: One hundred and ninety-two clinical isolates of $P$. aeruginosa were characterized using polymerase chain reaction (PCR) and 16S rDNA sequencing. Antibiotic resistance patterns were determined using the disk diffusion method, while the minimum inhibitory concentrations (MICs) of selected antibiotics against resistant isolates were determined by macro broth dilution and E-test strip methods. The resistant isolates were screened for the presence of PstS gene using PCR.

Results: Of 192 clinical isolates of P. aeruginosa, 136 (70.83\%) were resistant to at least two antibiotics. Of these, 135 (99\%) could be classified as multidrug-resistant $P$. aeruginosa (MDR-PA), 63 (46\%) were extensively drug-resistant (XDR-PA), while 38 (28\%) were pandrug-resistant (PDR-PA). The isolates exhibited high level of resistance to cefotaxime and ticarcillin, and low levels of resistance to meropenem and imipenem. The MIC values for meropenem against the resistant isolates were generally $<32 \mathrm{mg} / \mathrm{L}$, while the values for other antibiotics ranged from 32 to $>128 \mathrm{mg} / \mathrm{L}$. Multiple antibiotic resistance indexes of the MDR-PA ranged from 0.27 to 0.91 and the most prevalent pattern of resistance was Piperacillin ${ }^{\mathrm{R}}-$ Ticarcillin $^{\mathrm{R}}-$ Piperacillin/Tazobactam ${ }^{\mathrm{R}}-$ Cefotaxime $^{\mathrm{R}}-$ Ceftazidime $^{\mathrm{R}}-$ Gentamicn $^{\mathrm{R}}-$ Tobramycin $^{\mathrm{R}}-$ Ciprofloxacin $^{\mathrm{R}}$. About $50 \%$ of the resistant isolates possessed the PstS gene.

Conclusions: The results confirmed the presence of XDR, PDRPA, and PstS gene in P. aeruginosa strains. There is an urgent need for healthcare practitioners to address the problem of multidrug resistance, by implementing a more rational and appropriate use of antibiotics.

Keywords: Pseudomonas aeruginosa, Antimicrobial resistance, Multidrug-resistant Pseudomonas aeruginosa, Extensively drug-resistant, $P s t S$ gene.

(c) 2020 The Authors. Published by Innovare Academic Sciences Pvt Ltd. This is an open access article under the CC BY license (http://creativecommons. org/licenses/by/4. 0/) DOI: http://dx.doi.org/10.22159/ajpcr.2020.v13i4.36669

\section{INTRODUCTION}

Pseudomonas aeruginosa is a Gram-negative aerobic bacillus belonging to the Pseudomonadaceae family. It is highly ubiquitous in nature and may be found in most moist environments in the hospital, including sinks, cleaning buckets, drains, humidifiers, and toilet water [1]. $P$. aeruginosa is becoming an increasingly important cause of health care-associated infections [2-5]. It has been reported to be the sixth most common nosocomial pathogen overall and second most common pathogen in ventilator-associated pneumonia in US hospitals [4]. It has also been ranked second among Gram-negative pathogens reported to the United States National Nosocomial Infection Surveillance System [3]. P. aeruginosa constitutes a common pathogen in hospitals, particularly in intensive care units, due to its ubiquitous nature, ability to survive in moist environments, innate resistance to many antibiotics and antiseptics, and ability to acquire resistance to many drug classes $[3,6,7]$. It is a leading cause of septicemia, pneumonia, meningitis, wound, urinary tract, surgical wound, burn, and ear infections [8].

Resistance in Pseudomonas may be mediated through several distinct mechanisms, including the production of $\beta$-lactamases, efflux pumps, target site, and outer membrane modifications, but antimicrobial agents that have been found to be effective against $P$. aeruginosa include aminoglycosides (gentamicin, tobramycin, amikacin, and netilmicin); carbapenems (imipenem, meropenem, and doripenem); cephalosporins (ceftazidime and cefepime); fluoroquinolones (ciprofloxacin and levofloxacin); penicillins $\beta$-lactamase inhibitors (ticarcillin-clavulanic acid and piperacillin-tazobactam); monobactams (aztreonam); phosphonic acids (fosfomycin); and polymyxins (colistin and polymyxin B) $[9,10]$. Antibiotic resistant $P$. aeruginosa can be classified today Multidrug-resistant (MDR), extensively drug-resistant (XDR) or pandrug-resistant (PDR). MDR has been defined as a strain that develops resistance to at least one agent in three or more antimicrobial categories, XDR is a strain resistant to one agent in all categories except two or less, while PDR is a strain resistant to one agent in all categories $[9,11]$.

Of all the anti-Pseudomonas agents, the carbapenems have been widely used for empirical or directed therapy, whenever MDR P. aeruginosa is suspected. However, recent evidence indicates that resistance to the carbapenems is emerging in P. aeruginosa and improper use of these drugs could accelerate the occurrence [4,12]. This is a global concern, not just for Pseudomonas, but for other bacterial pathogens. However, for the developing world, especially, this concern appears to be more reality than speculation. The concern arises from certain practices observed in the developing world, including unregulated use/sale of antibiotics, over-prescription of antibiotics, release of antibiotics into wastewaters by drug manufacturers, poor sanitation, poor infection control, misuse of antibiotics, and among others [13]. These problems can, however, be solved by better surveillance and monitoring of drug resistance. To this effect, the World Health Organization (WHO) has proposed a new global surveillance network, although there are still questions about how the initiative will be funded [13].

Considering the constantly evolving pattern of antibiotic resistance in $P$. aeruginosa and high mortality rates associated with Pseudomonas infections, it is of great public health importance to continuously 
track the occurrence and spread of antibiotic resistance in this rapidly emerging superbug. In a WHO global map of antibiotic resistance data from 194 member countries, Nigeria was one of the countries for which no information was obtained [13].

Studies have reported that expression of the PstS gene enhances the virulence of MDR Pseudomonas strains [14]. PstS proteins are the cell-bound phosphate binding elements of the ubiquitous bacterial ATP-binding cassette phosphate uptake mechanisms. This study was, therefore, undertaken to determine the prevalence of MDR among clinical isolates of P. aeruginosa in Nsukka, Southeast Nigeria, determine the patterns of resistance and screen the isolates for the presence of $P s t S$ gene, which has been reported to confer increased virulence on MDR P. aeruginosa (MDRPA).

\section{METHODS}

\section{The bacterial strains}

A total of 192 strains of P. aeruginosa were collected from Microbiology Laboratories from various hospitals in Nsukka, from March 2016 to February 2017. Ethical approval and informed consent were not required by our Institution Ethics Committee because all bacterial isolates were collected, processed, and stored as part of routine diagnosis by the hospitals. No patient information was associated with the data. The isolates obtained from the various laboratories were subcultured onto Pseudomonas cetrimide agar (Oxoid, U.K) which was supplemented with $10 \mathrm{ml} / \mathrm{l}$ of glycerol and characterized using standard microbiological techniques such as grade like odor, pigment production, positive oxidase test, growth at $42^{\circ} \mathrm{C}$, and molecular method using $16 \mathrm{~S}$ rRNA primer targeting $P$. aeruginosa consensus region (Inqaba Biotechnical Company, South Africa)

\section{Antimicrobial susceptibility test}

One hundred and ninety-two P. aeruginosa isolates were used for in vitro susceptibility testing. Susceptibility to ten antibiotics, namely ticarcillin $75 \mu \mathrm{g}$; piperacillin $100 \mu \mathrm{g}$; piperacillin/tazobactam 100/10 $\mu \mathrm{g}$; ceftazidime $30 \mu \mathrm{g}$; cefotaxime $30 \mu \mathrm{g}$; imipenem $10 \mu \mathrm{g}$; meropenem $10 \mu \mathrm{g}$; gentamicin $10 \mu \mathrm{g}$; tobramycin $10 \mu \mathrm{g}$; and ciprofloxacin $5 \mu \mathrm{g}$ (Oxoid, UK) was evaluated on Muller-Hinton agar, using Kirby-Bauer disc diffusion method. The ten antibiotics represented six classes of antibiotic (Table 1). The isolates were considered susceptible or resistant according to the zone of inhibition recommended by the Clinical and Laboratory Standard Institute [15]. P. aeruginosa ATCC
27853 was used as the control strain. Isolates were considered MDR (MDRPA) if they showed resistance to three or more classes of the tested antibiotics.

\section{Multiple antibiotic resistance (MAR) index}

The MAR index was determined for each isolate by dividing the number of antibiotics to which the isolate is resistant by the total number of antibiotics tested [16].

$$
\text { MAR index }=\frac{\text { Number of antibioticsisolate is resistant to }}{\text { Totalnumber of antibiotics tested }}
$$

Determination of minimum inhibitory concentrations (MIC)

The MIC for the MDR P. aeruginosa was determined using macro broth dilution method and E-test method according to the CLSI standard [15]. $P$. aeruginosa isolates that were resistant to meropenem; ceftazidime; ciprofloxacin; and gentamicin were used for MIC study. Macro broth dilution was used to determine the MIC for ciprofloxacin and gentamicin while E-strip test was used for meropenem and ceftazidime.

\section{Genomic DNA extraction}

The genomic DNA was extracted from 20 MDR P. aeruginosa strain using Zymo research fungal/bacterial DNA Miniprep Kit (Zymo Research, USA) according to the manufacturer's protocol.

\section{DNA amplification}

The extracted DNA was amplified with 16S rRNA primer targeting $P$. aeruginosa consensus region and another primer targeting PstS gene that enhances the virulence of MDR Pseudomonas strains (Inqaba Biotechnical Company, South Africa), Table 2. The polymerase chain reaction (PCR) was carried out using the New England Biolabs one Taq 2X master mix with standard buffer. PCR reaction mixture was prepared in a $25 \mu \mathrm{l}$ reaction volume containing $12.5 \mu \mathrm{l}$ of $1 \mathrm{X}$ Master mix with standard buffer, $20 \mathrm{mM}$ Tris- $\mathrm{HCl}, 1.8 \mathrm{mM} \mathrm{MgCl}_{2}, 22 \mathrm{mM} \mathrm{NH}_{4} \mathrm{Cl}, 22 \mathrm{mM} \mathrm{KCl}, 0.2 \mathrm{mM}$ DNTPS, 5\% glycerol, 0.06\% GEPAL CA-630, 0.05\% Tween 20, 25 units $/ \mathrm{ml}$ Taq DNA polymerase (Biolab, England), $0.5 \mu \mathrm{l}(10 \mu \mathrm{M})$, each of the forward and reverse primers (Inqaba Biotechnical, South Africa), $5 \mu \mathrm{l}$ of the extracted DNA, and $6.5 \mu \mathrm{l}$ of sterile nuclease-free water (Norgen Biotek, Canada) to make up to $25 \mu \mathrm{l}$ of reaction volume. This was vortexed at low speed and placed in a thermal cycler machine (BIBBY) - Scientific Ltd., UK. The parameters for amplification were as follows initial denaturation of $94^{\circ} \mathrm{C}$ for $5 \mathrm{~min}$, followed by 35 amplification cycles of denaturation at

Table 1: Zone diameter interpretive standards and equivalent MIC breakpoints for Pseudomonas aeruginosa (Clinical and Laboratory Standard Institute, 2014)

\begin{tabular}{|c|c|c|c|c|c|c|c|}
\hline \multirow[t]{2}{*}{ Antimicrobial class } & \multirow{2}{*}{$\begin{array}{l}\text { Antimicrobial agent and disk } \\
\text { concentration }\end{array}$} & \multicolumn{3}{|c|}{ Zone diameter (mm) } & \multicolumn{3}{|c|}{ Equivalent MIC break pointing ( $\mathrm{ug} / \mathrm{ml}$ ) } \\
\hline & & $\mathbf{R}$ & I & $\mathbf{S}$ & $\mathbf{R}$ & I & $\mathbf{S}$ \\
\hline \multirow[t]{2}{*}{ Penicillins } & Ticarcillin $75 \mu \mathrm{g}$ & $\leq 15$ & $16-23$ & $\geq 24$ & $\geq 128$ & & $\leq 64$ \\
\hline & Piperacillin $100 \mu \mathrm{g}$ & $\leq 14$ & $15-20$ & $\geq 21$ & $\geq 128$ & & $\leq 64$ \\
\hline$\beta$-lactam $/ \beta$-lactamase inhibitor & Piperacillin-tazobactam $100 / 10 \mu \mathrm{g}$ & $\leq 14$ & $15-20$ & $\geq 21$ & $\geq 128 / 4$ & & $\leq 64 / 4$ \\
\hline \multirow[t]{2}{*}{ Cephems } & Ceftazidime $30 \mu \mathrm{g}$ & $\leq 14$ & $15-17$ & $\geq 18$ & $\geq 32$ & 16 & $\leq 8$ \\
\hline & Cefotaxime $30 \mu \mathrm{g}$ & $\leq 14$ & $15-17$ & $\geq 21$ & $\geq 64$ & & $\leq 8$ \\
\hline \multirow[t]{2}{*}{ Carbapenems } & Imipenem $10 \mu \mathrm{g}$ & $\leq 15$ & $16-18$ & $\geq 19$ & $\geq 16$ & & $\leq 4$ \\
\hline & Meropenem $10 \mu \mathrm{g}$ & $\leq 15$ & $16-18$ & $\geq 19$ & $\geq 16$ & & $\leq 4$ \\
\hline \multirow[t]{2}{*}{ Aminoglycosides } & Gentamicin $10 \mu \mathrm{g}$ & $\leq 12$ & $13-14$ & $\geq 15$ & $\geq 8$ & & $\leq 4$ \\
\hline & Tobramycin $10 \mu \mathrm{g}$ & $\leq 12$ & $13-14$ & $\geq 15$ & $\geq 8$ & & $\leq 4$ \\
\hline Fluoroquinolones & Ciprofloxacin $5 \mu \mathrm{g}$ & $\leq 15$ & $16-20$ & $\geq 21$ & $\geq 4$ & & $\leq 1$ \\
\hline
\end{tabular}

R: Resistant, 1: Intermediate, S: Sensitive, MIC: Minimal inhibiting concentration

Table 2: Primers used for Identification of Pseudomonas aeruginosa and detection of PstS gene

\begin{tabular}{|c|c|c|c|c|}
\hline Target gene & Function & Sequence $\left(5^{1---3^{1}}\right)$ & Amplicon size (pb) & Accession No: \\
\hline 16S rRNA & Consensus region & $\begin{array}{l}\text { AGAGTTTGATCCTGGCTCAG } \\
\text { ACGGCTACCTTGTTACGACTT }\end{array}$ & 1499 & HM045838 \\
\hline PstS & Enhances the virulence of MDR & $\begin{array}{l}\text { GGCTTTCGAGCAGAAGTACG } \\
\text { ATGTAGCCGTCCTTGACCAC }\end{array}$ & 606 & EF601159 \\
\hline
\end{tabular}


Table 3: Resistance patterns of multidrug-resistant strains of Pseudomonas aeruginosa

\begin{tabular}{|c|c|c|}
\hline Resistance pattern & $\begin{array}{l}\text { Classification } \\
\text { of resistance }\end{array}$ & $\begin{array}{l}\text { No. of } \\
\text { isolates }\end{array}$ \\
\hline $\begin{array}{l}\mathrm{PRL}^{\mathrm{R}}, \mathrm{TIC}^{\mathrm{R}}, \mathrm{TZP}^{\mathrm{R}}, \mathrm{CTX}^{\mathrm{R}}, \mathrm{CAZ}^{\mathrm{R}}, \mathrm{MEM}^{\mathrm{R}}, \mathrm{IMP}^{\mathrm{R}}, \\
\mathrm{CN}^{\mathrm{R}}, \mathrm{TOB}^{\mathrm{R}}, \mathrm{CIP}^{\mathrm{R}}\end{array}$ & PDR & 4 \\
\hline $\begin{array}{l}\mathrm{PRL}^{\mathrm{R}}, \mathrm{TZP}^{\mathrm{R}}, \mathrm{TIC}^{\mathrm{R}}, \mathrm{CTX}^{\mathrm{R}}, \mathrm{CAZ}^{\mathrm{R}}, \mathrm{MEM}^{\mathrm{R}}, \mathrm{CN}^{\mathrm{R}}, \\
\mathrm{TOB}^{\mathrm{R}}, \mathrm{CIP}^{\mathrm{R}}\end{array}$ & PDR & 1 \\
\hline $\begin{array}{l}\mathrm{PRL}^{\mathrm{R}}, \mathrm{TIC}^{\mathrm{R}}, \mathrm{TZP}^{\mathrm{R}}, \mathrm{CTX}^{\mathrm{R}}, \mathrm{CAZ}^{\mathrm{R}}, \mathrm{MEM}^{\mathrm{R}}, \mathrm{IMP}^{\mathrm{R}}, \\
\mathrm{CN}^{\mathrm{R}}, \mathrm{TOB}^{\mathrm{R}}\end{array}$ & PDR & 2 \\
\hline $\begin{array}{l}\mathrm{PRL}^{\mathrm{R}}, \mathrm{TIC}^{\mathrm{R}}, \mathrm{TZP}^{\mathrm{R}}, \mathrm{CTX}^{\mathrm{R}}, \mathrm{CAZ}^{\mathrm{R}}, \mathrm{MEM}^{\mathrm{R}}, \mathrm{CN}^{\mathrm{R}}, \\
\mathrm{TOB}^{\mathrm{R}}, \mathrm{CIP}^{\mathrm{R}}\end{array}$ & PDR & 16 \\
\hline $\begin{array}{l}\mathrm{PRL}^{\mathrm{R}}, \mathrm{TIC}^{\mathrm{R}}, \mathrm{TZP}^{\mathrm{R}}, \mathrm{CTX}^{\mathrm{R}}, \mathrm{CAZ}^{\mathrm{R}}, \mathrm{IMP}^{\mathrm{R}}, \mathrm{CN}^{\mathrm{R}}, \\
\mathrm{TOB}^{\mathrm{R}}, \mathrm{CIP}^{\mathrm{R}}\end{array}$ & PDR & 3 \\
\hline $\begin{array}{l}\mathrm{PRL}^{\mathrm{R}}, \mathrm{gTIC}^{\mathrm{R}}, \mathrm{TZP}^{\mathrm{R}}, \mathrm{CTX}^{\mathrm{R}}, \mathrm{CAZ}^{\mathrm{R}}, \mathrm{CN}^{\mathrm{R}}, \mathrm{TOB}^{\mathrm{R}}, \\
\mathrm{CIP}^{\mathrm{R}}\end{array}$ & XDR & 2 \\
\hline $\begin{array}{l}\mathrm{PRL}^{\mathrm{R}}, \mathrm{TIC}^{\mathrm{R}}, \mathrm{CTX}^{\mathrm{R}}, \mathrm{CAZ}^{\mathrm{R}}, \mathrm{MEM}^{\mathrm{R}}, \mathrm{CN}^{\mathrm{R}}, \mathrm{TOB}^{\mathrm{R}}, \\
\mathrm{CIP}^{\mathrm{R}}\end{array}$ & XDR & 2 \\
\hline $\begin{array}{l}\mathrm{PRL}^{\mathrm{R}}, \mathrm{TIC}^{\mathrm{R}}, \mathrm{TZP}^{\mathrm{R}}, \mathrm{CTX}^{\mathrm{R}}, \mathrm{CAZ}^{\mathrm{R}}, \mathrm{MEM}^{\mathrm{R}}, \mathrm{CN}^{\mathrm{R}}, \\
\mathrm{CIP}^{\mathrm{R}}\end{array}$ & PDR & 1 \\
\hline $\begin{array}{l}\mathrm{PRL}^{\mathrm{R}}, \mathrm{TIC}^{\mathrm{R}}, \mathrm{TZP}^{\mathrm{R}}, \mathrm{CTX}^{\mathrm{R}}, \mathrm{CAZ}^{\mathrm{R}}, \mathrm{CN}^{\mathrm{R}}, \mathrm{TOB}^{\mathrm{R}}, \\
\mathrm{CIP}^{\mathrm{R}}\end{array}$ & XDR & 17 \\
\hline $\begin{array}{l}\mathrm{PRL}^{\mathrm{R}}, \mathrm{TIC}^{\mathrm{R}}, \mathrm{TZP}^{\mathrm{R}}, \mathrm{CTX}^{\mathrm{R}}, \mathrm{CAZ}^{\mathrm{R}}, \mathrm{MEM}^{\mathrm{R}}, \mathrm{IMP}^{\mathrm{R}}, \\
\mathrm{CIP}^{\mathrm{R}}\end{array}$ & XDR & 1 \\
\hline $\begin{array}{l}\mathrm{PRL}^{\mathrm{R}}, \mathrm{TIC}^{\mathrm{R}}, \mathrm{TZP}^{\mathrm{R}}, \mathrm{CTX}^{\mathrm{R}}, \mathrm{MEM}^{\mathrm{R}}, \mathrm{CN}^{\mathrm{R}}, \mathrm{TOB}^{\mathrm{R}}, \\
\mathrm{CIP}^{\mathrm{R}}\end{array}$ & PDR & 1 \\
\hline $\begin{array}{l}\mathrm{PRL}^{\mathrm{R}}, \mathrm{TIC}^{\mathrm{R}}, \mathrm{TZP}^{\mathrm{R}}, \mathrm{CTX}^{\mathrm{R}}, \mathrm{IMP}^{\mathrm{R}}, \mathrm{CN}^{\mathrm{R}}, \mathrm{TOB}^{\mathrm{R}}, \\
\mathrm{CIP}^{\mathrm{R}}\end{array}$ & PDR & 1 \\
\hline $\begin{array}{l}\mathrm{PPL}^{\mathrm{R}}, \mathrm{TIC}^{\mathrm{R}}, \mathrm{CTX}^{\mathrm{R}}, \mathrm{CAZ}^{\mathrm{R}}, \mathrm{CN}^{\mathrm{R}}, \mathrm{TOB}^{\mathrm{R}}, \mathrm{CIP}^{\mathrm{R}}, \\
\mathrm{MEM}^{\mathrm{R}}\end{array}$ & XDR & 1 \\
\hline $\begin{array}{l}\mathrm{TIC}^{\mathrm{R}}, \mathrm{TZP}^{\mathrm{R}}, \mathrm{CTX}^{\mathrm{R}}, \mathrm{CAZ}^{\mathrm{R}}, \mathrm{MEM}^{\mathrm{R}}, \mathrm{CN}^{\mathrm{R}}, \mathrm{TOB}^{\mathrm{R}} \text {, } \\
\mathrm{CIP}^{\mathrm{R}}\end{array}$ & PDR & 1 \\
\hline $\begin{array}{l}\mathrm{PRL}^{\mathrm{R}}, \mathrm{TIC}^{\mathrm{R}}, \mathrm{CTX}^{\mathrm{R}}, \mathrm{CAZ}^{\mathrm{R}}, \mathrm{MEM}^{\mathrm{R}}, \mathrm{IMP}^{\mathrm{R}}, \mathrm{CN}^{\mathrm{R}}, \\
\mathrm{TOB}^{\mathrm{R}}\end{array}$ & XDR & 1 \\
\hline $\mathrm{TIC}^{\mathrm{R}}, \mathrm{CTX}^{\mathrm{R}}, \mathrm{CAZ}^{\mathrm{R}}, \mathrm{MEM}^{\mathrm{R}}, \mathrm{CN}^{\mathrm{R}}, \mathrm{TOB}^{\mathrm{R}}, \mathrm{CIP}^{\mathrm{R}}$ & XDR & 1 \\
\hline $\mathrm{TIC}^{\mathrm{R}}, \mathrm{TZP}^{\mathrm{R}}, \mathrm{CTX}^{\mathrm{R}}, \mathrm{CAZ}^{\mathrm{R}}, \mathrm{CN}^{\mathrm{R}}, \mathrm{TOB}^{\mathrm{R}}, \mathrm{CIP}^{\mathrm{R}}$ & XDR & 1 \\
\hline $\mathrm{PRL}^{\mathrm{R}}, \mathrm{TIC}^{\mathrm{R}}, \mathrm{TZP}^{\mathrm{R}}, \mathrm{CTX}^{\mathrm{R}}, \mathrm{CAZ}^{\mathrm{R}}, \mathrm{CN}^{\mathrm{R}}, \mathrm{CIP}^{\mathrm{R}}$ & XDR & 1 \\
\hline $\mathrm{PRL}^{\mathrm{R}}, \mathrm{TIC}^{\mathrm{R}}, \mathrm{CTX}^{\mathrm{R}}, \mathrm{CAZ}^{\mathrm{R}}, \mathrm{IMP}^{\mathrm{R}}, \mathrm{CN}^{\mathrm{R}}, \mathrm{TOB}^{\mathrm{R}}$ & XDR & 1 \\
\hline $\mathrm{PRL}^{\mathrm{R}}, \mathrm{TIC}^{\mathrm{R}}, \mathrm{TZP}^{\mathrm{R}}, \mathrm{CTX}^{\mathrm{R}}, \mathrm{CAZ}^{\mathrm{R}}, \mathrm{IMP}^{\mathrm{R}}, \mathrm{TOB}^{\mathrm{R}}$ & XDR & 1 \\
\hline $\mathrm{PRL}^{\mathrm{R}}, \mathrm{TIC}^{\mathrm{R}}, \mathrm{TZP}^{\mathrm{R}}, \mathrm{CTX}^{\mathrm{R}}, \mathrm{CN}^{\mathrm{R}}, \mathrm{TOB}^{\mathrm{R}}, \mathrm{CIP}^{\mathrm{R}}$ & XDR & 1 \\
\hline $\mathrm{PRL}^{\mathrm{R}}, \mathrm{TIC}^{\mathrm{R}}, \mathrm{CTX}^{\mathrm{R}}, \mathrm{CAZ}^{\mathrm{R}}, \mathrm{CN}^{\mathrm{R}}, \mathrm{TOB}^{\mathrm{R}}, \mathrm{CIP}^{\mathrm{R}}$ & XDR & 7 \\
\hline $\mathrm{PRL}^{\mathrm{R}}, \mathrm{TIC}^{\mathrm{R}}, \mathrm{TZP}^{\mathrm{R}}, \mathrm{CTX}^{\mathrm{R}}, \mathrm{CAZ}^{\mathrm{R}}, \mathrm{MEM}^{\mathrm{R}}, \mathrm{TOB}^{\mathrm{R}}$ & XDR & 2 \\
\hline $\mathrm{TIC}^{\mathrm{R}}, \mathrm{TZP}^{\mathrm{R}}, \mathrm{CTX}^{\mathrm{R}}, \mathrm{CAZ}^{\mathrm{R}}, \mathrm{TOB}^{\mathrm{R}}, \mathrm{CIP}^{\mathrm{R}}, \mathrm{CN}^{\mathrm{R}}$ & XDR & 2 \\
\hline $\mathrm{PRL}^{\mathrm{R}}, \mathrm{TIC}^{\mathrm{R}}, \mathrm{TZP}^{\mathrm{R}}, \mathrm{CTX}^{\mathrm{R}}, \mathrm{CAZ}^{\mathrm{R}}, \mathrm{CN}^{\mathrm{R}}, \mathrm{CIP}^{\mathrm{R}}$ & XDR & 4 \\
\hline $\mathrm{PRL}^{\mathrm{R}}, \mathrm{TIC}^{\mathrm{R}}, \mathrm{TZP}^{\mathrm{R}}, \mathrm{CTX}^{\mathrm{R}}, \mathrm{IMP}^{\mathrm{R}}, \mathrm{CN}^{\mathrm{R}}, \mathrm{CIP}^{\mathrm{R}}$ & PDR & 2 \\
\hline $\mathrm{PRL}^{\mathrm{R}}, \mathrm{TIC}^{\mathrm{R}}, \mathrm{TZP}^{\mathrm{R}}, \mathrm{CTX}^{\mathrm{R}}, \mathrm{CAZ}^{\mathrm{R}}, \mathrm{MEM}^{\mathrm{R}}, \mathrm{CIP}^{\mathrm{R}}$ & XDR & 2 \\
\hline $\mathrm{TIC}^{\mathrm{R}}, \mathrm{CTX}^{\mathrm{R}}, \mathrm{CAZ}^{\mathrm{R}}, \mathrm{CN}^{\mathrm{R}}, \mathrm{TOB}^{\mathrm{R}}, \mathrm{CIP}^{\mathrm{R}}, \mathrm{MEM}^{\mathrm{R}}$ & XDR & 3 \\
\hline $\mathrm{PRL}^{\mathrm{R}}, \mathrm{TIC}^{\mathrm{R}}, \mathrm{TZP}^{\mathrm{R}}, \mathrm{CTX}^{\mathrm{R}}, \mathrm{IMP}^{\mathrm{R}}, \mathrm{CIP}^{\mathrm{R}}$ & XDR & 1 \\
\hline $\mathrm{PRL}^{\mathrm{R}}, \mathrm{TIC}^{\mathrm{R}}, \mathrm{CTX}^{\mathrm{R}}, \mathrm{MEM}^{\mathrm{R}}, \mathrm{CN}^{\mathrm{R}}, \mathrm{TOB}^{\mathrm{R}}$ & XDR & 2 \\
\hline $\mathrm{PRL}^{\mathrm{R}}, \mathrm{TIC}^{\mathrm{R}}, \mathrm{TZP}^{\mathrm{R}}, \mathrm{CTX}^{\mathrm{R}}, \mathrm{CAZ}^{\mathrm{R}}, \mathrm{CN}^{\mathrm{R}}$ & XDR & 7 \\
\hline $\mathrm{TIC}^{\mathrm{R}}, \mathrm{CTX}^{\mathrm{R}}, \mathrm{CAZ}^{\mathrm{R}}, \mathrm{CN}^{\mathrm{R}}, \mathrm{TOB}^{\mathrm{R}}, \mathrm{CIP}^{\mathrm{R}}$ & XDR & 3 \\
\hline $\mathrm{PRL}^{\mathrm{R}}, \mathrm{TIC}^{\mathrm{R}}, \mathrm{CTX}^{\mathrm{R}}, \mathrm{CN}^{\mathrm{R}}, \mathrm{TOB}^{\mathrm{R}}, \mathrm{CIP}^{\mathrm{R}}$ & XDR & 2 \\
\hline $\mathrm{PRL}^{\mathrm{R}}, \mathrm{TIC}^{\mathrm{R}}, \mathrm{TZP}^{\mathrm{R}}, \mathrm{CTX}^{\mathrm{R}}, \mathrm{CAZ}^{\mathrm{R}}, \mathrm{CIP}^{\mathrm{R}}$ & XDR & 2 \\
\hline $\mathrm{PRL}^{\mathrm{R}}, \mathrm{TIC}^{\mathrm{R}}, \mathrm{CTX}^{\mathrm{R}}, \mathrm{CAZ}^{\mathrm{R}}, \mathrm{CN}^{\mathrm{R}}, \mathrm{TOB}^{\mathrm{R}}$ & MDR & 1 \\
\hline $\mathrm{PRL}^{\mathrm{R}}, \mathrm{TIC}^{\mathrm{R}}, \mathrm{TZP}^{\mathrm{R}}, \mathrm{CTX}^{\mathrm{R}}, \mathrm{CAZ}^{\mathrm{R}}, \mathrm{TOB}^{\mathrm{R}}$ & XDR & 1 \\
\hline $\mathrm{PRL}^{\mathrm{R}}, \mathrm{TIC}^{\mathrm{R}}, \mathrm{TZP}^{\mathrm{R}}, \mathrm{CTX}^{\mathrm{R}}, \mathrm{CAZ}^{\mathrm{R}}$ & MDR & 4 \\
\hline $\mathrm{TIC}^{\mathrm{R}}, \mathrm{CTX}^{\mathrm{R}}, \mathrm{CAZ}^{\mathrm{R}}, \mathrm{CN}^{\mathrm{R}}, \mathrm{CIP}^{\mathrm{R}}$ & MDR & 1 \\
\hline $\mathrm{TIC}^{\mathrm{R}}, \mathrm{CTX}^{\mathrm{R}}, \mathrm{CAZ}^{\mathrm{R}}, \mathrm{CN}^{\mathrm{R}}, \mathrm{TOB}^{\mathrm{R}}$ & MDR & 4 \\
\hline $\mathrm{TIC}^{\mathrm{R}}, \mathrm{CTX}^{\mathrm{R}}, \mathrm{CAZ}^{\mathrm{R}}, \mathrm{TOB}^{\mathrm{R}}, \mathrm{CIP}^{\mathrm{R}}$ & MDR & 1 \\
\hline $\mathrm{PRL}^{\mathrm{R}}, \mathrm{TIC}^{\mathrm{R}}, \mathrm{TZP}^{\mathrm{R}}, \mathrm{CTX}^{\mathrm{R}}, \mathrm{CN}^{\mathrm{R}}$ & MDR & 1 \\
\hline $\mathrm{TIC}^{\mathrm{R}}, \mathrm{CTX}^{\mathrm{R}}, \mathrm{CN}^{\mathrm{R}}, \mathrm{TOB}^{\mathrm{R}}$ & MDR & 1 \\
\hline $\mathrm{PRL}^{\mathrm{R}}, \mathrm{TIC}^{\mathrm{R}}, \mathrm{TZP}^{\mathrm{R}}, \mathrm{CIP}^{\mathrm{R}}$ & MDR & 1 \\
\hline $\mathrm{PRL}^{\mathrm{R}}, \mathrm{TZP}^{\mathrm{R}}, \mathrm{CTX}^{\mathrm{R}}, \mathrm{TOB}^{\mathrm{R}}$ & MDR & 1 \\
\hline $\mathrm{TIC}^{\mathrm{R}}, \mathrm{TZP}^{\mathrm{R}}, \mathrm{CTX}^{\mathrm{R}}, \mathrm{CAZ}^{\mathrm{R}}$ & MDR & 1 \\
\hline $\mathrm{TIC}^{\mathrm{R}}, \mathrm{CTX}^{\mathrm{R}}, \mathrm{CN}^{\mathrm{R}}, \mathrm{TOB}^{\mathrm{R}}$ & MDR & 3 \\
\hline $\mathrm{TIC}^{\mathrm{R}}, \mathrm{CTX}^{\mathrm{R}}, \mathrm{CAZ}^{\mathrm{R}}, \mathrm{CN}^{\mathrm{R}}$ & MDR & 3 \\
\hline $\mathrm{TIC}^{\mathrm{R}}, \mathrm{CTX}^{\mathrm{R}}, \mathrm{CAZ}^{\mathrm{R}}, \mathrm{MEM}^{\mathrm{R}}$ & MDR & 8 \\
\hline $\mathrm{PRL}^{\mathrm{R}}, \mathrm{TZP}^{\mathrm{R}}, \mathrm{CTX}^{\mathrm{R}}$ & MDR & 1 \\
\hline $\mathrm{TIC}^{\mathrm{R}}, \mathrm{CTX}^{\mathrm{R}}, \mathrm{CAZ}^{\mathrm{R}}$ & & 1 \\
\hline $\mathrm{PRL}^{\mathrm{R}}, \mathrm{TIC}^{\mathrm{R}}, \mathrm{CTX}^{\mathrm{R}}$ & & 2 \\
\hline
\end{tabular}

Table 3: (Continued)

\begin{tabular}{lll}
\hline Resistance pattern & $\begin{array}{l}\text { Classification } \\
\text { of resistance }\end{array}$ & $\begin{array}{l}\text { No. of } \\
\text { isolates }\end{array}$ \\
\hline $\mathrm{TIC}^{\mathrm{R}}, \mathrm{CTX}^{\mathrm{R}}, \mathrm{CN}^{\mathrm{R}}$ & $\mathrm{MDR}$ & 1 \\
$\mathrm{TIC}^{\mathrm{R}}, \mathrm{CTX}^{\mathrm{R}}, \mathrm{CAZ}^{\mathrm{R}}$ & & 1 \\
\hline
\end{tabular}

PRL $^{\mathrm{R}}$ : Piperacillin resistant, TZP ${ }^{\mathrm{R}}$ : Piperacillin/Tazobactam resistant, TIC $^{\mathrm{R}}$ : Ticarcillin resistant, CTX: Cefotaxime resistant, CAZ: Ceftazidime resistant, MEM $^{\mathrm{R}}$ : Meropenem resistant, IMPR: Imipenem resistant, TOB $^{\mathrm{R}}$ : Tobramycin resistant, $\mathrm{CN}^{\mathrm{R}}$ : Gentamicin resistant, CIPR: Ciprofloxacin resistant, MDR: Multidrug-resistant, XDR: Extensively drug-resistant, PDR: Pandrug-resistant

Table 4: MAR indexes of Pseudomonas aeruginosa isolates

\begin{tabular}{lll}
\hline MAR index & $\begin{array}{l}\text { No. of resistant Pseudomonas } \\
\text { aeruginosa isolates }\end{array}$ & $\begin{array}{l}\text { \% Prevalence } \\
\text { of MAR }\end{array}$ \\
\hline 0.27 & 18 & 13.23 \\
0.36 & 20 & 14.71 \\
0.45 & 16 & 11.76 \\
0.55 & 17 & 12.50 \\
0.64 & 20 & 14.71 \\
0.73 & 25 & 18.38 \\
0.82 & 19 & 13.97 \\
0.91 & 1 & 0.74 \\
\hline
\end{tabular}

MAR: Multiple antibiotic resistance

$94^{\circ} \mathrm{C}$ for $1 \mathrm{~min}$, annealing at $50^{\circ} \mathrm{C}$ and $55^{\circ} \mathrm{C}$ for $1 \mathrm{~min}$ for $16 \mathrm{~S}$ rRNA and PstS primers, and extension at $72^{\circ} \mathrm{C}$ for $1 \mathrm{~min}$. This was followed by a final extension step of $5 \mathrm{~min}$ at $72^{\circ} \mathrm{C}$. The amplification products (amplicons) were separated on $1.5 \%$ agarose gel stained with ethidium bromide and electrophoresis was carried out at 70 volts for $90 \mathrm{~min}$ and visualized/ illuminated under ultraviolet transilluminator. A 100 bp DNA ladder (Norgen Biotek Corp., Canada) was used as DNA molecular weight marker.

Data analysis

Frequency of MDRPA and percentage of resistance to antibiotics were calculated.

\section{RESULTS}

\section{Antimicrobial sensitivity}

Of 192 isolates of $P$. aeruginosa that were tested, 136 (70.83\%) were resistant to at least two antibiotics. The resistance of the isolates against a panel of 10 antibiotics was cefotaxime, 88.02\%; ticarcillin, 87.50\%; ceftazidime, 64.06\%; ciprofloxacin, $62.50 \%$; piperacillin, $58.33 \%$; tobramycin, 57.29\%; gentamicin, 56.25\%; piperacillin/tazobactam, 55.73\%; meropenem, 24.48\%; and imipenem, $12.50 \%$. Evaluation of resistance of the isolates to different classes of antibiotics revealed that $135(99 \%)$ were resistant to at least one antibiotic in three different classes (MDR-PA); 63 (46\%) were resistant to all except two or three classes (XDR-PA), while 38 (28\%) were resistant to all six classes of antibiotics (PDR-PA), as shown in Table 3. The most prevalent pattern of resistance was $\left(\mathrm{PRL}^{\mathrm{R}} \mathrm{TIC}^{\mathrm{R}} \mathrm{TZP}^{\mathrm{R}} \mathrm{CTX}^{\mathrm{R}} \mathrm{CAZ}^{\mathrm{R}} \mathrm{CN}^{\mathrm{R}} \mathrm{TOB}^{\mathrm{R}} \mathrm{CIP}^{\mathrm{R}}\right)$.

\section{Analysis of MAR index}

Analysis of the MAR index showed that the isolates had MAR indexes ranging from 0.27 to 0.91 (Table 4) while evaluation of the MIC of some of the antibiotics against the isolates ranged from 0.12 to $128 \mu \mathrm{g} / \mathrm{ml}$ (Table 5).

\section{Occurrence of PstS gene}

The result of the PCR revealed that of 20 MDRPA isolates that were screened for PstS gene, lane 1, 3-7, 9, 10, 15, and 16 showed positive amplification of the $606 \mathrm{bp}$ PstS gene (Fig. 1).

\section{DISCUSSION}

In this study, 192 clinical isolates of $P$. aeruginosa were screened against a panel of ten antibiotics, representing six classes of antibiotics. 
Table 5: The MIC and MBC $(\mu \mathrm{g} / \mathrm{ml})$ of multidrug resistant Pseudomonas aeruginosa

\begin{tabular}{|c|c|c|c|c|}
\hline \multirow[t]{2}{*}{ Organisms } & \multicolumn{4}{|l|}{ MIC $(\mu \mathrm{g} / \mathrm{ml})$} \\
\hline & $\begin{array}{l}\text { Meropenem } \\
(\mathrm{S} \leq 4 \mu \mathrm{g}, \mathrm{I}=8 \mu \mathrm{g} / \mathrm{ml} \\
\text { and } \mathrm{R} \leq 16(\mu \mathrm{g} / \mathrm{ml})^{*}\end{array}$ & $\begin{array}{l}\text { Ceftazidime } \\
(\mathrm{S} \leq 8, \mathrm{I}=16 \mu \mathrm{g} / \mathrm{ml} \\
\text { and } \mathrm{R} \geq 32 \mu \mathrm{g} / \mathrm{ml})^{*}\end{array}$ & $\begin{array}{l}\text { Ciprofloxacin } \\
(\mathrm{S} \leq 1 \mu \mathrm{g} / \mathrm{ml}, \mathrm{I}=2 \\
\text { and } \mathrm{R} \geq 4 \mu \mathrm{g} / \mathrm{ml})^{*}\end{array}$ & $\begin{array}{l}\text { Gentamicin } \\
(\mathrm{S} \leq 4 \mu \mathrm{g} / \mathrm{ml}, \mathrm{I}=8 \mu \mathrm{g} / \mathrm{ml} \\
\text { and } \mathrm{R} \geq 16 \mu \mathrm{g} / \mathrm{ml})^{*}\end{array}$ \\
\hline ATCC 27853 & 0.25 & 1.00 & 2.00 & 4.00 \\
\hline 1 & 8.00 & 4.00 & 32.00 & 16.00 \\
\hline 2 & 0.12 & 2.00 & 64.00 & 64.00 \\
\hline 3 & 0.50 & 64.00 & 32.00 & 16.00 \\
\hline 4 & 1.00 & 16.00 & 16.00 & 8.00 \\
\hline 5 & 8.00 & 32.00 & 128.00 & 32.00 \\
\hline 6 & 0.50 & 4.00 & 32.00 & 16.00 \\
\hline 7 & 2.00 & 16.00 & 16.00 & 8.00 \\
\hline 8 & 8.00 & 64.00 & 64.00 & 32.00 \\
\hline 9 & 32.00 & 8.00 & 128.00 & 64.00 \\
\hline 10 & 2.00 & 32.00 & 8.00 & 16.00 \\
\hline
\end{tabular}

Grey shade indicates resistant strains. ${ }^{*}$ CLSI breakpoints. S: Sensitive, I: Intermediate, R: Resistant, MIC: Minimal inhibiting concentration, MBC: Minimal bactericidal concentration, CLSI: Clinical and Laboratory Standard Institute

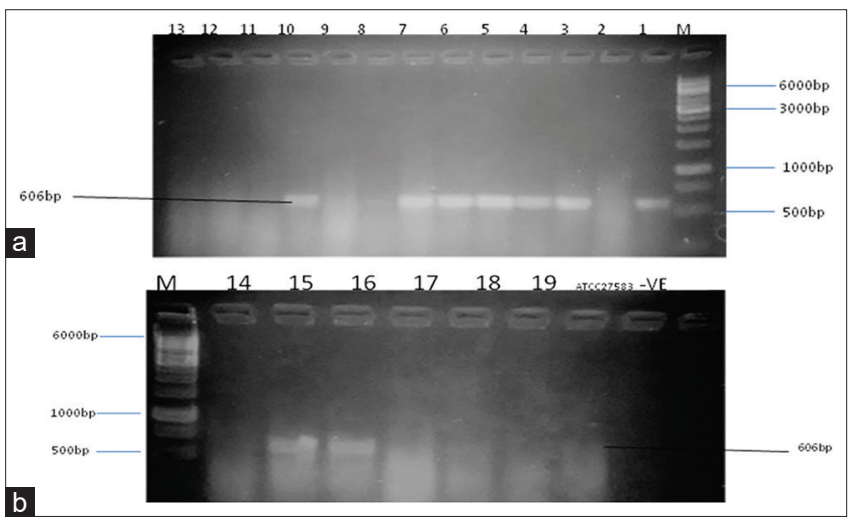

Fig. 1: (a and b) Polymerase chain reaction detection of 606 bp amplicons of $P$ stS primer for identification of multidrug-resistant Pseudomonas aeruginosa, Lane M shows bands for $1 \mathrm{~kb}(1000 \mathrm{bp})$ molecular weight standard. Lanes $1,3-7,9,10,15$, and 16 show a positive amplification band. Indicating the presence of $P$ stS gene in $P$. aeruginosa isolates analyzed. Lanes $14,17,18,19$, and ATCC 27583 show negative amplification and produced no visible band

The highest levels of resistance were recorded to the cephalosporin (cefotaxime and ceftazidime) and $\beta$-lactam (ticarcillin) antibiotics, while the lowest resistance levels were recorded to the carbapenems (meropenem and imipenem). P. aeruginosa is known to be intrinsically resistant to many antimicrobial agents including $\beta$-lactams, the older quinolones, chloramphenicol, tetracycline, macrolides, trimethoprimsulfamethoxazole, and rifampin $[10,16]$. Until recently, the most important antipseudomonal agents were some $\beta$-lactams (ticarcillin and piperacillin), cephalosporins (cefoperazone, ceftazidime, and cefotaxime), aminoglycosides (gentamicin, tobramycin, and amikacin), fluoroquinolones (notably ciprofloxacin), and $\beta$-lactam- $\beta$-lactamase inhibitor combinations $[3,10]$. Recently, however, there are increasing reports of emerging resistance to these antipseudomonal agents in clinical settings, worldwide [3,10,17-20]. In this study, P. aeruginosa isolates exhibited high levels of resistance to different antibiotics used. Although fluoroquinolones are regarded as a good option in many parts of the world, in this study, the P. aeruginosa isolates exhibited high levels of resistance to this class of antibiotics.

The introduction of carbapenems into clinical practice represented a great advance for the treatment of serious bacterialinfections caused by $\beta$-lactam resistant bacteria and they have been reported by many surveillance studies to be the drug of choice for MDR-P. aeruginosa [4,10,12,21]. In this study, the carbapenems (meropenem and imipenem) were found to be among the most active agents.
Although many studies have reported low resistance or outright $100 \%$ sensitivity for imipenem and meropenem [22-29], 12.5\% and 24.5\% resistance were recorded for both drugs, respectively, in this study, which is a cause for concern. It is also worrisome that about $46 \%$ of the clinical isolates in this study have gone beyond exhibiting mere MDR to extensive drug-resistance (XDR), while up to $28 \%$ may now be classified as PDR, as shown in Table 3. The most prevalent pattern of resistance among the isolates was $\mathrm{PRL}^{\mathrm{R}}-\mathrm{TIC}^{\mathrm{R}}-\mathrm{TZP}^{\mathrm{R}}-\mathrm{CTX}^{\mathrm{R}}-\mathrm{CAZ}^{\mathrm{R}}-\mathrm{CN}^{\mathrm{R}}-\mathrm{TOB}^{\mathrm{R}}-\mathrm{CIP}^{\mathrm{R}}$.

It is evident from these findings that a serious public health problem already exists in the environment under study, as a significant number of isolates is exhibiting resistance not only to the classical antipseudomonal drugs but also to the $\beta$-lactam- $\beta$-lactamase inhibitor combination and the last line carbapenem group. This can only indicate that this environment would be experiencing a high degree of treatment failure. The high degree of resistance recorded in this study is in agreement with a 2012 study in Southwest Nigeria [30]. It has been suggested that this trend may be due to selective pressure from the overuse and misuse of antibiotics, prevalent in the country.

Some authors have suggested that the MAR index may give an indirect indication of the probable source of an organism, an index of greater than 0.2 , indicating that an organism must have originated from an environment where antibiotics are often used [16,31]. As can be seen in Table 4, all the MDR (XDR and PDR inclusive) isolates had MAR indexes ranging from 0.27 to 0.91 . These observations tend to validate the assertion by some authors that antibiotic resistance is sweeping the developing world [13] and point to the need for tighter restrictions on the sale/use of antibiotics if the rapid spread of resistance is to be checked.

Studies have reported that expression of the PstS gene enhances the virulence of MDR Pseudomonas strains. The gene encodes a periplasmic phosphate-binding protein, which, when present, confers a highly virulent phenotype on MDR strains of $P$. aeruginosa. In addition, the development of MDR may be related to the overproduction of the PstS gene [14]. About $50 \%$ of the isolates in this study possessed the PstS gene, which further raises alarm about the impending public health crises due to P. aeruginosa in Nsukka, Southeast Nigeria.

\section{CONCLUSIONS}

There is the presence of XDR, PDRPA, and PstS gene in P. aeruginosa strains in Southeast Nigeria. The findings from this study show an alarming degree of resistance among $P$. aeruginosa isolates in Nsukka, Nigeria and an urgent need for healthcare practitioners and policymakers to address the problem of MDR by implementing a more rational and appropriate use of antibiotics. Establishment of an effective surveillance program and strict disinfection policy in hospital 
environments would also help to control the spread of MDR, XDR, and PDRPA in hospital settings.

\section{ACKNOWLEDGMENT}

The authors wish to thank the generous assistance of the doctors and nurses of the different hospitals used for their cooperation, understanding, and assistance when this research was going on. The authors gratefully acknowledge the microbiology department of the various hospitals for willingly providing the $P$. aeruginosa clinical isolates used in this study.

\section{AUTHORS' CONTRIBUTIONS}

Martina C Agbo, as the corresponding author, carried out the experiments and drafted the manuscript. Ifeoma M Ezeonu conceived, organized, and supervised this research work and reviews the manuscript. Anthony C Ike helped in review of the work, offered advice, participated in PCR, and read through the manuscript. Celestina C Ugwu assisted during the sample collection and helped in some bench work.

\section{CONFLICTS OF INTEREST}

The authors declare that there are no conflicts of interest.

\section{FUNDING}

The authors declare that they did not have any funding source or grant to support their research work.

\section{REFERENCES}

1. Jefferies JM, Cooper T, Yam T, Clarke SC. Pseudomonas aeruginosa outbreaks in the neonatal intensive care unit-a systematic review of risk factors and environmental sources. J Med Microbiol 2012;61:1052-61.

2. Hirsch EB, Tam VH. Impact of multidrug-resistant Pseudomonas aeruginosa infection on patient outcomes. Expert Rev Pharmacoecon Outcomes Res 2010;10:441-51.

3. Alnour TM, Ahmed-Abakur EH. Multidrug resistant Pseudomonas aeruginosa: Medical impact, pathogenicity, resistance mechanisms and epidemiology. JSM Microbiol 2017;5:1046.

4. Nguyen L, Garcia J, Gruenberg K, MacDougall C. Multidrug-resistant Pseudomonas Infections: Hard to treat, but hope on the horizon? Curr Infect Dis Rep 2018;20:23.

5. Palavutitotai N, Jitmuang A, Tongsai S, Kiratisin P, Angkasekwinai N. Epidemiology and risk factors of extensively drug-resistant Pseudomonas aeruginosa infections. PLoS One 2018;13:e193431.

6. Porras-Gómez M, Vega-Baudrit J, SNúñez-Corrales S. Overview of multidrug-resistant Pseudomonas aeruginosa and novel therapeutic approaches. J Biomater Nanobiotechnol 2012;3:519-27.

7. Mahmoud $\mathrm{AB}$, Zahran WA, Hindawi GR, Labib AZ, Galal R. Prevalence of multidrug-resistant Pseudomonas aeruginosa in patients with nosocomial infections at a University hospital in Egypt, with special reference to typing methods. J Virol Microbiol 2013;2013:1-13.

8. Todar K. Pseudomonas aeruginasa. In: Online Textbook for Bacteriology. Madison, WI: Kenneth Todar, University of WisconsinMadison Department of Bacteriology; 2008.

9. Magiorakos AP, Srinivasan A, Carey RB, Carmeli Y, Falagas ME, Giske CG, et al. Multidrug-resistant, extensively drug-resistant and pandrug-resistant bacteria: An international expert proposal for interim standard definitions for acquired resistance. Clin Microbiol Infect 2012;18:268-81.

10. Rossolini GM, Mantengoli E. Treatment and control of severe infections caused by multiresistant Pseudomonas aeruginosa. Clin Microbiol Infect 2005;11 Suppl 4:17-32.

11. Inacio HS, Bomfim MR, França RO, Farias LM, Carvalho MA, Serufo JC, et al. Phenotypic and genotypic diversity of multidrugresistant Pseudomonas aeruginosa isolates from bloodstream infections recovered in the hospitals of Belo Horizonte, Brazil. Chemotherapy
2014;60:54-62.

12. Barrios CC, Ciancotti-Oliver L, Bautista-Rentero D, Adán-Tomás C, Zanón-Viguer V. A new treatment choice against multi-drug resistant Pseudomonas aeruginosa: Doripenem. J Bacteriol Parasitol 2014;5:199.

13. Reardon S. Antibiotic resistance sweeping developing world. Nature 2014;509:141-2.

14. Zaborina O, Holbrook C, Chen Y, Long J, Zaborin A, Morozova I, et al. Structure-function aspects of PstS in multi-drug-resistant Pseudomonas aeruginosa. PLoS Pathog 2008;4:e43.

15. Clinical and Laboratory Standards Institute. Performance Standards for Antimicrobial Susceptibility Testing: Twenty-fourth Informational Supplement. Vol. 34. United States: Clinical and Laboratory Standards Institute; 2014. p. M100-S24

16. Krumperman PH. Multiple antibiotic resistance indexing of Escherichia coli to identify high-risk sources of fecal contamination of foods. Appl Environ Microbiol 1983;46:165-70.

17. Mesaros N, Nordmann P, Plésiat P, Roussel-Delvallez M, Van Eldere J, Glupczynski Y, et al. Pseudomonas aeruginosa: Resistance and therapeutic options at the turn of the new millennium. Clin Microbiol Infect 2007;13:560-78

18. Paranjothi S, Dheepa R. Screening for multidrug resistance Bacteria Pseudomonas aeruginosa in hospitalized patient in Hosur, Krishnagiri (DT). Int J Pharm Bio Sci 2010;1:1-15.

19. Senthamarai S, Reddy AS, Sivasankari S, Anitha C, Somasunder V, Kumudhavathi MS, et al. Resistance pattern of Pseudomonas aeruginosa in a tertiary care hospital of Kanchipuram, Tamilnadu, India. J Clin Diagn Res 2014;8:DC30-2.

20. Ghanbarzadeh Corehtash Z, Khorshidi A, Firoozeh F, Akbari H, Mahmoudi Aznaveh A. Biofilm formation and virulence factors among Pseudomonas aeruginosa isolated from burn patients. Jundishapur $\mathbf{J}$ Microbiol 2015;8:e22345.

21. Thomas BT, Agu GC, Makanjuola SO, Davies A. Plasmid profiling and antibiotic resistance of extended spectrum beta lactamases producing Pseudomonas aeruginosa expressing AMPC beta-lactamase enzyme. Am Eurasian J Sci Res 2015;10:109-117.

22. Mendiratta DK, Deotale V, Narang P. Metallo-beta-lactamase producing Pseudomonas aeruginosa in a hospital from a rural area. Indian J Med Res 2005;121:701-3.

23. Bashir D, Thakar MA, Fomda BA, Bash G, Zahoor D, Ahmad SA, et al. Detection of metallobeta-lactamases (MBL) producing Pseudomonas aeruginosa at a tertiary care hospital in Kashmir. Afr J Microbiol Res 2011;5:164-72.

24. Tripathi P, Banerjee G, Saxena S, Gupta MK, Ramteke PW. Antibiotic resistance pattern of Pseudomonas aeruginosa isolated from patients of lower respiratory tract infection. Afr J Microbiol Res 2011;5:2955-9.

25. Xiao H, Ye X, Liu Q, Li L. Antibiotic susceptibility and genotype patterns of Pseudomonas aeruginosa from mechanical ventilation-associated pneumonia in intensive care units. Biomed Rep 2013;1:589-93.

26. Jayanthi S, Jeya M. Plasmid profile analysis and bla VIM gene detection of metalo $\beta$-lactamase (MBL) producing Pseudomonas aeruginosa isolates from clinical samples. J Clin Diagn Res 2014;8:DC16-9.

27. Shaikh S, Fatima J, Shakil S, Danish Rizvi SM, Kamal MA. Prevalence of multidrug resistant and extended spectrum beta-lactamase producing Pseudomonas aeruginosa in a tertiary care hospital. Saudi J Biol Sci 2015;22:62-4

28. Raafat MM, Ali-Tamma M, Ali AE. Phenotypic and genotypic characterization of Pseudomonas aeruginosa isolates from Egyptian hospital. Afr J Microbiol Res 2016;10:1645-53.

29. Ranjbar R, Owlia P, Saderi H, Mansouri S, Jonaidi-Jafari N, Izadi M, et al. Characterization of Pseudomonas aeruginosa strains isolated from burned patients hospitalized in a major burn center in Tehran, Iran. Acta Med Iran 2011;49:675-9.

30. Akingbade OA, Balogun SA, Ojo DA, Afolabi RO, Motaya BO, Okerentugbe PO, et al. Plasmid profile analysis of multi-drug resistant Pseudomonas aeruginosa isolated from wound infections in South West Nigeria. World Appl Sci J 2012;20:766-775.

31. Paul S, Bezbaruah RL, Roy MK, Ghosh AC. Multiple antibiotic resistance (MAR) index and its reversion in Pseudomonas aeruginosa. Lett Appl Microbiol 1997;24:169-71. 